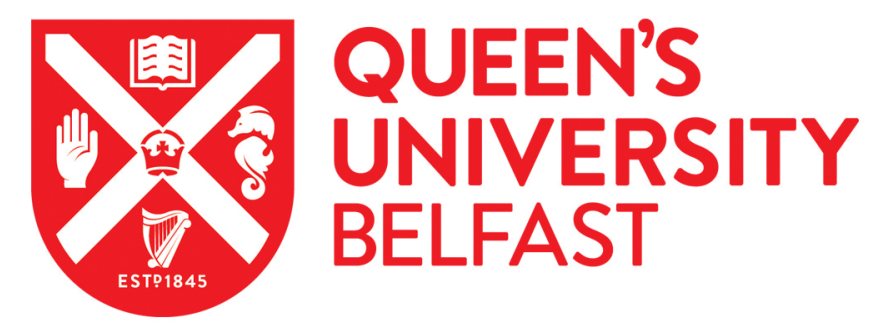

\title{
Correlation Effects in the Compton Profile of Silicon
}

Kralik, B., Delaney, P., \& Louie, S. G. (1998). Correlation Effects in the Compton Profile of Silicon. Physical Review Letters, 80(19), 4253-4256. https://doi.org/10.1103/PhysRevLett.80.4253

\section{Published in:}

Physical Review Letters

Queen's University Belfast - Research Portal:

Link to publication record in Queen's University Belfast Research Portal

\section{General rights}

Copyright for the publications made accessible via the Queen's University Belfast Research Portal is retained by the author(s) and / or other copyright owners and it is a condition of accessing these publications that users recognise and abide by the legal requirements associated with these rights.

Take down policy

The Research Portal is Queen's institutional repository that provides access to Queen's research output. Every effort has been made to ensure that content in the Research Portal does not infringe any person's rights, or applicable UK laws. If you discover content in the Research Portal that you believe breaches copyright or violates any law, please contact openaccess@qub.ac.uk. 


\title{
Correlation Effects in the Compton Profile of Silicon
}

\author{
Balázs Králik, Paul Delaney, and Steven G. Louie \\ Department of Physics, University of California at Berkeley, Berkeley, California 94720, \\ and Materials Science Division, Ernest Orlando Lawrence Berkeley National Laboratory, Berkeley, California 94720
}

(Received 27 June 1997; revised manuscript received 15 January 1998)

\begin{abstract}
$A b$ initio nonlocal pseudopotential variational quantum Monte Carlo techniques are used to compute the correlation effects on the valence momentum density and Compton profile of silicon. Our results for this case are in excellent agreement with the Lam-Platzman correction computed within the local density approximation. Within the approximations used, we rule out valence electron correlations as the dominant source of discrepancies between calculated and measured Compton profiles of silicon. [S0031-9007(98)06062-1]
\end{abstract}

PACS numbers: 71.55.Cn, 71.15.Ap, 71.15.Hx, 71.15.Th

The Compton effect [1] has been a very early test of the predictions of the quantum theory of solids [2]. With the advent of new, very high resolution Compton spectroscopy [3-8] there is renewed interest in this experimental technique as a probe of the detailed electronic structure of real materials [9].

Crystalline silicon, as one of the technologically most important and best studied materials, is an ideal test system to consider. Silicon momentum densities and Compton profiles have been studied experimentally with various techniques such as synchrotron radiation $\mathrm{x}$-ray scattering $[10,11], \gamma$-ray scattering [12-16], and angular correlation of positron annihilation [17-19]. There have also been many theoretical computations of these quantities, ranging in complexity from tight-binding [20], empirical pseudopotential [21] to ab initio pseudopotential [22] and allelectron methods [23,24]. All calculations to date have relied on single-particle band theory in their interpretation of momentum densities and Compton profiles.

While the existing theoretical calculations are in close agreement with experimental observations, discrepancies remain to be explained. In particular, it is found that theoretical Compton profiles are too high at $\mathbf{q}=0$ as compared to experiment in materials ranging from lithium [7] to silicon [9]. One possible source for such discrepancies is electron correlation beyond the single-particle approximation. In this Letter we develop the nonlocal pseudopotential variational quantum Monte Carlo (VQMC) method for momentum densities, and apply it to study the electron correlation effects on the Compton profile of silicon.

In the VQMC method, the true many-body state is approximated by

$$
\Psi=\Psi_{J} d^{\dagger} d^{\downarrow},
$$

where the $d$ are Slater determinants of single-particle orbitals $\phi_{i}$ such as the eigenstates of the Kohn-Sham equations $[25,26]$. We generate the single-particle orbitals by performing density functional theory (DFT) calculations in the local density approximation (LDA), using the PerdewZunger [27] parametrization of the Ceperley-Alder [28] exchange-correlation potential. Correlation effects are included into our many-body wave function via the Jastrow factor $\Psi_{J}$. The exact form of the Jastrow factor is determined variationally. Following Fahy, Wang, and Louie [29], we only include single- and two-particle terms:

$$
\Psi_{J}\left(\mathbf{r}_{1}, \ldots, \mathbf{r}_{N}\right)=\exp \left(\sum_{i} \chi\left(\mathbf{r}_{i}\right)-\sum_{i<j} u\left(\left|\mathbf{r}_{i}-\mathbf{r}_{j}\right|\right)\right) .
$$

The two-particle term $u$ incorporates the correlations resulting from the Coulomb repulsion between electrons. It consists of two terms, $u_{\sigma}=u_{\sigma}^{P}+u_{\sigma}^{\mathrm{var}}$. The $\sigma$ index takes the values $\sigma=\uparrow \uparrow$ or $\uparrow \downarrow$, i.e., a different twoparticle term applies between parallel and antiparallel spins. The usual plasmon term $u_{\sigma}^{P}(r)=\frac{A}{r}\left[1-\exp \left(\frac{r}{F_{\sigma}}\right)\right]$ is constructed to have the correct asymptotic dependence at $\mathbf{r} \rightarrow \infty$ and to satisfy the cusp condition at $\mathbf{r} \rightarrow 0$. $A$ is treated as a single variational parameter in this term [29].

We introduce further variational freedom into the twoparticle term via $u^{\mathrm{var}}$. $u^{\mathrm{var}}$ is the most general polynomial expression on the interval $\left[0, L_{0}\right]$ that satisfies the conditions (1) $u^{\prime}(0)=0$, (2) $u\left(L_{0}\right)=0$, and (3) $u^{\prime}\left(L_{0}\right)=0$ [30]. The first condition ensures that the cusp condition remains satisfied. Conditions (2) and (3) ensure that $u$ and its derivative remain continuous at the cutoff radius $L_{0}$. This is necessary to avoid singularities in the kinetic energy of the wave function. The cutoff radius $L_{0}$ is chosen to be large enough so that the variational freedom in $u^{\mathrm{var}}$ can effectively influence the shape of the Coulomb hole. This means that $L_{0}$ should be chosen to be on the order of a few average electron-electron separations. Since the average $r_{s}$ in silicon is $2, L_{0}$ was chosen to be 15 a.u. We have used polynomials up to order 12 , which corresponds to 18 variational parameters in this term.

As the two-particle term $u$ has the effect of making the electrons avoid each other beyond what is dictated by the antisymmetry of the wave function, the $u$ term alone tends to smooth the charge density [29]. The singleparticle part $\chi$ of the Jastrow factor introduces variational freedom that allows adjustment of the charge density to further minimize the energy or the variance of the energy. 
In our study we used the $\chi$ term in two ways: First, $\chi$ can be treated as a fully variational part of the wave function with no constraints. We used up to nine parameters (nine independent Fourier coefficients) in our study. This variational $\chi$-factor will be referred to as $\chi^{\text {var }}$ in the following.

Second, $\chi$ can be used to restore the DFT charge density following the prescription of Ref. [29]. In the rest of this Letter the notation $\chi^{\mathrm{CD}}$ will refer to this choice. In this case, $\chi$ is chosen to be $\chi^{\mathrm{CD}}(\mathbf{r})=\frac{1}{2} C \ln \left(\frac{\rho_{\mathrm{LDA}}(\mathbf{r})}{\rho_{\text {uonly }}(\mathbf{r})}\right)$, where $C$ is treated as a single variational parameter.

The optimal parameters in the Jastrow factor are determined via minimizing the variance of the total energy [30]. We estimate the variance of the total energy as

$$
\sigma^{2}=\frac{1}{\sum_{j} w\left(\mathbf{R}_{j}\right)} \sum_{i} \frac{\left(\hat{H}-E_{T}\right)^{2} \Psi\left(\mathbf{R}_{i}\right)}{\Psi\left(\mathbf{R}_{i}\right)} w\left(\mathbf{R}_{i}\right),
$$

where $\hat{H}$ is the Hamiltonian operator, and $\left\{\mathbf{R}_{i}\right\}$ is a set of $N$-electron configurations having probability distribution $\left|\Psi_{\text {guide }}\left(\mathbf{R}_{i}\right)\right|^{2} . \quad \Psi_{\text {guide }}$ is our best estimate of the optimal wave function and $E_{T}$ is our best estimate for the groundstate energy. The weighting factors

$$
w\left(\mathbf{R}_{i}\right)=\frac{\left|\Psi\left(\mathbf{R}_{i}\right)\right|^{2}}{\left|\Psi_{\text {guide }}\left(\mathbf{R}_{i}\right)\right|^{2}}
$$

are required because the $\mathbf{R}_{i}$ are distributed with probability density $\left|\Psi_{\text {guide }}\left(\mathbf{R}_{i}\right)\right|^{2}$ and not with probability density $\left|\Psi\left(\mathbf{R}_{i}\right)\right|^{2}$. This formalism allows us to minimize $\sigma^{2}$ with respect to the variational parameters in the Jastrow factor on a fixed set of configurations, thus eliminating statistical noise from the minimization procedure. In practice, the variance minimization is carried out iteratively, i.e., the $\left\{\mathbf{R}_{i}\right\}$ are regenerated several times. The variance minimization and all subsequent calculations are carried out in a 16-atom (64-electron) simulation cell.

Table I shows the effect of introducing variational freedom in the wave function on the total energy. While most of the energy gain is realized with the simple 1-parameter plasmon term $u^{P}$ [29], the introduction of further variational freedom lowers the energy further appreciably. Similar gains have been reported for Ge by Williamson et al. [30].

Once the optimal parameters have been determined, we turn to calculating the momentum density. The

TABLE I. Effect of the variational freedom in the Jastrow factor on the total energy of Si. The no-Jastrow-factor energy was set to zero. In parentheses are the estimates of the statistical error in the last digit.

\begin{tabular}{lc}
\hline \hline Jastrow factor & Energy gain $(\mathrm{eV} /$ atom $)$ \\
\hline None & $0.00(7)$ \\
$u^{P}$ & $-3.10(5)$ \\
$u^{P}+\chi^{\mathrm{CD}}$ & $-3.23(4)$ \\
$u^{P}+\chi^{\mathrm{var}}$ & $-3.45(4)$ \\
$u^{P}+u^{\mathrm{var}}+\chi^{\mathrm{var}}$ & $-3.59(4)$ \\
\hline \hline
\end{tabular}

momentum density for a many-body system is given by the expression

$$
\begin{aligned}
N(\mathbf{k})= & \int d \mathbf{s} d \mathbf{r}_{1} \cdots d \mathbf{r}_{N_{e}} \\
& \times \Psi^{*}\left(\mathbf{r}_{1} \cdots \mathbf{r}_{N_{e}}\right) e^{i \mathbf{k} \cdot \mathbf{s}} \Psi\left(\mathbf{r}_{1}+\mathbf{s} \cdots \mathbf{r}_{N_{e}}\right), \\
= & \left(N_{\text {conf }}\right)^{-1} \sum_{i}^{N_{\text {conf }}} \int d \mathbf{s} e^{i \mathbf{k} \cdot \mathbf{s}} \Psi\left(\mathbf{R}_{i}+\mathbf{s}\right) / \Psi\left(\mathbf{R}_{i}\right),
\end{aligned}
$$

where the $\left\{\mathbf{R}_{i}\right\}$ are configurations generated with probability density $\left|\Psi\left(\mathbf{R}_{i}\right)\right|^{2}$. At each configuration the remaining three-dimensional integration is carried out directly on a uniform, randomly shifted grid. In our study this uniform grid was chosen to have $3 \times 3 \times 3$ points. Our method mirrors that of Ref. [31] used to sample pair correlation functions.

In general, converged momentum distributions require large $k$-point grids for the LDA calculation [22]. A dense $k$-grid corresponds to a large supercell simulation. Since doing a large supercell calculation is computationally prohibitive within VQMC, we do small supercell calculations with generalized periodic boundary conditions. In this scheme, the many-body wave function is required to have the property

$$
\frac{\Psi\left(\mathbf{r}_{1}, \ldots, \mathbf{r}_{i}+\mathbf{L}, \ldots, \mathbf{r}_{N_{e}}\right)}{\Psi\left(\mathbf{r}_{1}, \ldots, \mathbf{r}_{i}, \ldots, \mathbf{r}_{N_{e}}\right)}=e^{i \mathbf{K} \cdot \mathbf{L}}
$$

where $\mathbf{L}$ is a lattice vector of the simulation cell and the phase factor $\Phi=e^{i \mathbf{K} \cdot \mathbf{L}}$ is independent of the index of the electron which is being moved. This property is guaranteed if the single-particle orbitals $\phi_{i}$ that form the Slater determinants in the many-body wave function all satisfy the same boundary condition $\phi_{i}(\mathbf{r}+\mathbf{L})=$ $e^{i \mathbf{k} \cdot \mathbf{L}} \phi(\mathbf{r})$. In other words, the constituent single-particle wave functions all have to correspond to the same $k$ point of the Brillouin zone of the simulation cell. In the case where the simulation cell is a $K \times L \times M$ replica of the primitive cell, the constituent single-particle wave functions have to fall on a (possibly shifted) $K \times L \times M$ Monkhorst-Pack [32] grid.

We are able to compute momentum densities for a dense $k$-point grid by repeating the VQMC calculation with several shifted sets of LDA wave functions. With our $2 \times 2 \times 2$ simulation cell, ten calculations, each with a different set of wave functions, were required to obtain a $10 \times 10 \times 10 k$-point resolution. At the same time, we can calculate the momentum density due to just the LDA orbitals, with no Jastrow factor.

Figure 1 shows the effect of the quality of the wave function on the correlation correction to the LDA momentum densities $N_{\mathrm{VQMC}}(\mathbf{k})-N_{\mathrm{LDA}}(\mathbf{k})$ at selected values of $\mathbf{k}$. The wave function only containing the plasmon term $u^{P}$ yields a clearly incorrect correction to $N_{\mathrm{LDA}}(\mathbf{k})$. In contrast, the 2-parameter form $\left(u=u^{P}+\chi^{\mathrm{CD}}\right)$ first used by Fahy, Wang, and Louie [29] and our best 28-parameter show identical (within statistical error) shifts of weight 


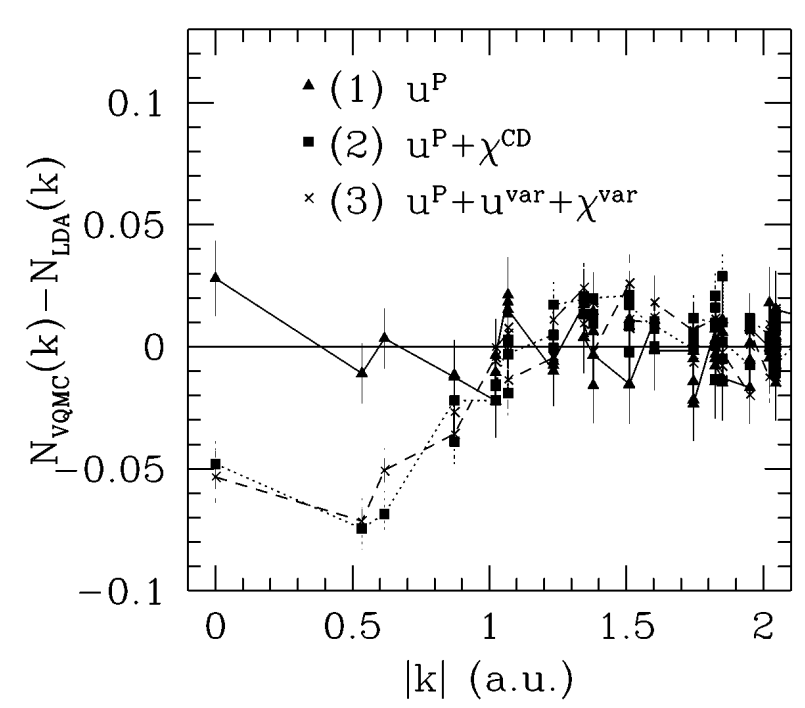

FIG. 1. Momentum density difference between VQMC and LDA calculations with three wave functions in (electrons/primitive cell)/(a.u. $)^{3}$ (see text). The graph summarizes data for various directions of $\mathbf{k}$. The lines connecting points are a guide to the eye. Expected statistical errors are indicated by vertical bars.

from low to high momentum values. By analogy to the homogeneous electron gas, we do expect such a weight transfer. The weight transfer is most marked at $|\mathbf{k}|<1$ a.u., where the momentum density is reduced by approximately $2 \%$ of its value at $\mathbf{k}=0$. The correlation effect is isotropic within the achieved error bars.

In order to compare our calculations to experimental observations, we integrate our momentum densities to obtain Compton profiles. Recall that in the impulse approximation $[33,34]$ the Compton profile is given as a twodimensional integral of the momentum density,

$$
J(\mathbf{q})=\int d \mathbf{k}^{\prime} N\left(\mathbf{k}^{\prime}\right) \delta\left(\hat{\mathbf{q}} \cdot \mathbf{k}^{\prime}-q\right) .
$$

In Fig. 2 we plot an experimental valence Compton profile [9] and the results of an $a b$ initio DFT-LDA calculation for the valence Compton profile along the (100) direction in silicon, as well as the difference $\Delta J(q)$ between these two curves. The calculation is performed within the pseudopotential approximation and the resulting pseudowave-functions are reconstructed to regain oscillations in the core regions as described in [22]. The LDA curve agrees closely with other theoretical predictions $[23,24]$. Figure 3 shows the correlation correction to the directional profiles in three high-symmetry directions. Correlations beyond the DFT-LDA cause weight to shift from lower to higher momenta. The correction is isotropic within the achieved error bars. The reduction of weight at $\mathbf{k}=0$ corresponds to approximately $1 \%$ of the $\mathbf{k}=0$ magnitude of the valence Compton profile. We find that correlation corrections are responsible for about $25 \%$ of the difference between the best theoretical calculations (Refs. [22-24]) of the valence Compton profile and experiment.

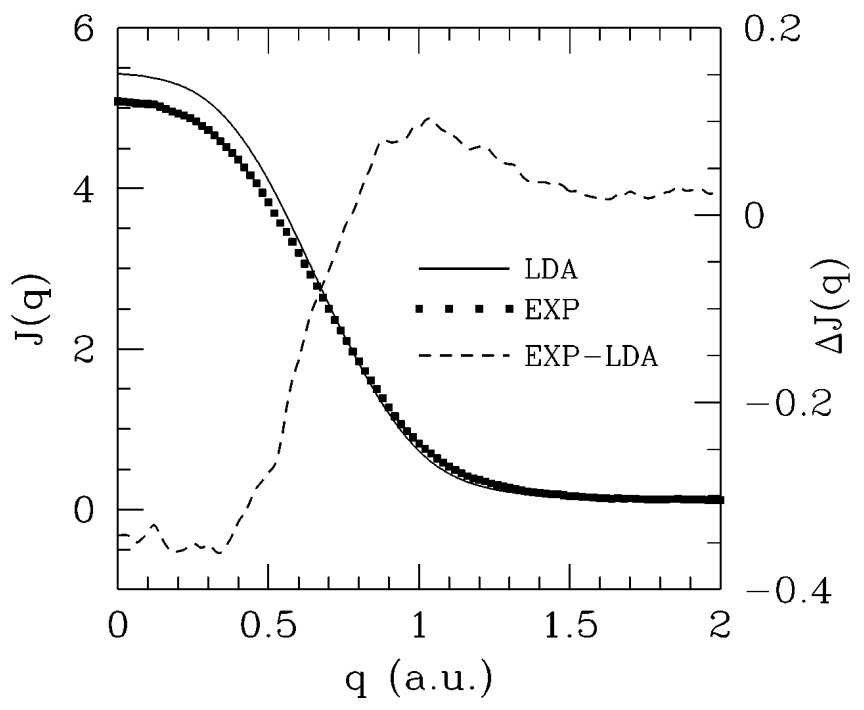

FIG. 2. Comparison between experimental and DFT-LDA valence Compton profiles along the (100) direction in silicon. The left vertical axis gives the scale of the two Compton profiles, and the right vertical axis applies to the difference $\Delta J(q)=J_{\mathrm{EXP}}(q)-J_{\mathrm{LDA}}(q)$.

The standard method to include correlation effects beyond the single-particle picture into momentum density and Compton profile calculations has been the LamPlatzman correction [35] in the LDA. This correction to the momentum density is, in principle, exact in the framework of density functional theory, but is approximate and isotropic in the LDA. We computed the Lam-Platzman correction to silicon Compton profiles within the LDA using diffusion Monte Carlo data for the homogeneous

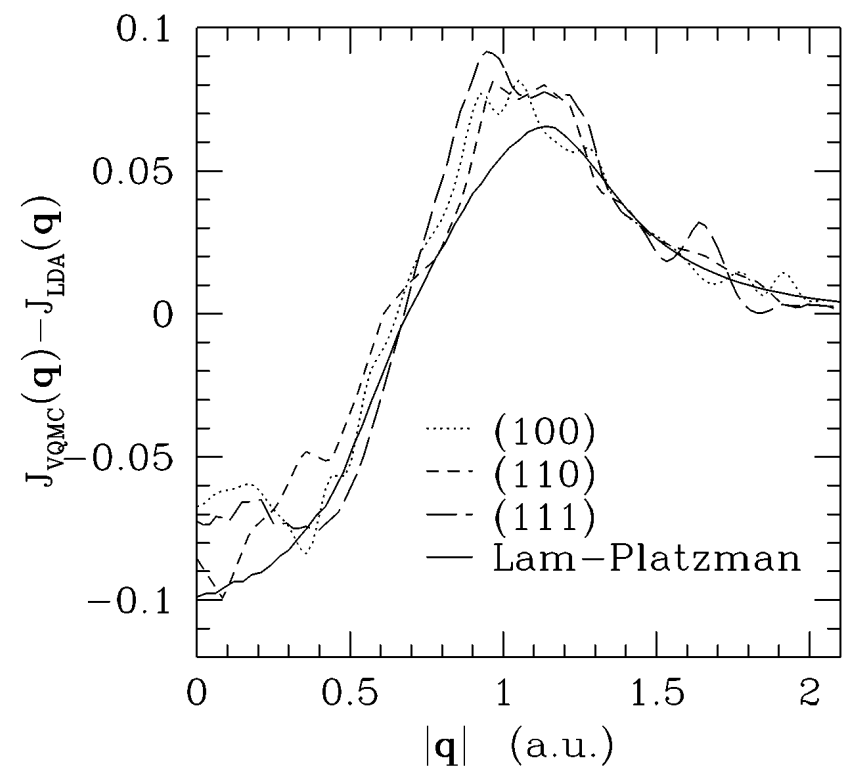

FIG. 3. Correlation correction to the LDA Compton profile of silicon. The standard error associated with the VQMC data is 0.01 . 
electron gas given by Ortiz and Ballone [36]. The momentum density corrections were then integrated to yield correlation corrections to the Compton profiles. Figure 3 shows the Lam-Platzman correction along with our VQMC results, which may depend on direction. The two computations are in quite good agreement.

In conclusion, we computed, for the first time, the VQMC correlation correction to the valence Compton profile of silicon. We have shown that, although sophisticated variational wave functions are necessary to obtain a good estimate of the ground-state energy of silicon, the correct momentum density is already obtained with the simple 2-parameter variational wave function first used by Fahy, Wang, and Louie [29] for solids. Our results show that valence correlation effects can be ruled out as the dominant source of discrepancy between experimentally observed and calculated Compton profiles of silicon. This indicates that some other nontrivial physics must be present for which the Compton profile acts as a sensitive probe. These effects could include the failure of the impulse approximation, core-core interaction effects, experimental uncertainty, or multiple-scattering corrections.

It is further found that the Lam-Platzman correction predicts this correction accurately for $\mathrm{Si}$. We expect that correlation corrections to momentum densities and Compton profiles of systems whose ground-state properties are well described within the LDA, such as simple metals, will be similarly well described by the Lam-Platzman correction. It remains an interesting open question as to what happens for systems where the LDA is not adequate. Momentum densities in, for example, transition metal oxides could exhibit novel features not captured by the LDA and the Lam-Platzman correction.

One of us (B. K.) gratefully acknowledges Dr. Jeffrey Grossman, Dr. Andrew Williamson, and Dr. Lubos Mitas for many helpful discussions on Monte Carlo methods. This work was supported by National Science Foundation Grant No. DMR-9520554 and by the Director, Office of Energy Research, Office of Basic Energy Sciences, Materials Sciences Division of the U.S. Department of Energy under Contract No. DE-AC03-76SF00098. Computer time was provided by the NSF at the Cornell Theory Center, and Cray T3E parallel computer time was provided by the Department of Energy at NERSC, Lawrence Berkeley National Laboratory.

[1] B. Williams, Compton Scattering (McGraw-Hill, New York, 1976).

[2] J. W. M. Du Mond, Phys. Rev. 33, 643 (1925).
[3] G. Loupias, J. Petiau, A. Issolah, and M. Schneider, Phys. Status Solidi B 102, 79 (1980).

[4] Y. Sakurai et al., Rev. Sci. Instrum. 63, 1190 (1992).

[5] A. Berthold et al., Nucl. Instrum. Methods Phys. Res., Sect. A 317, 373 (1992).

[6] C. Blaas et al., Phys. Rev. Lett. 75, 1984 (1995).

[7] Y. Sakurai et al., Phys. Rev. Lett. 74, 2252 (1995).

[8] K. Hamalainen et al., Phys. Rev. B 54, 5453 (1996).

[9] Informal Proceedings of the Second International Workshop on Compton Scattering and Fermiology, Tokyo, Japan, 1995 (unpublished).

[10] N. Shiotani et al., J. Phys. Condens. Matter 1, SA27 (1989).

[11] N. Sakai et al., J. Phys. Soc. Jpn. 58, 3270 (1989).

[12] W. A. Reed and P. Eisenberger, Phys. Rev. B 6, 4596 (1972).

[13] W. Schulke, Phys. Status Solidi B 62, 453 (1974).

[14] P. Pattison and J. R. Schneider, Solid State Commun. 28, 581 (1978).

[15] P. Pattison, N. K. Hansen, and J. R. Schneider, Chem. Phys. 59, 231 (1981).

[16] N. K. Hansen, P. Pattison, and J. R. Schneider, Z. Phys. B 66, 305 (1987).

[17] J.C. Erskine and J.D. McGervey, Phys. Rev. 151, 615 (1966).

[18] K. Fujiwara and T. Hyodo, J. Phys. Soc. Jpn. 35, 1133 (1973).

[19] G. Dlubek and W. Brummer, Phys. Status Solidi B 73, K107 (1976).

[20] A. Seth and D. E. Ellis, J. Phys. C 10, 181 (1977).

[21] H. Nara, K. Shindo, and T. Kobayashi, J. Phys. Soc. Jpn. 46, 79 (1979).

[22] P. Delaney, B. Kralik, and S. G. Louie, Phys. Rev. B (to be published).

[23] A. Bansil, 1995 (unpublished).

[24] C. Blaas, 1995 (unpublished).

[25] P. Hohenberg and W. Kohn, Phys. Rev. 136, B864 (1964).

[26] W. Kohn and L. J. Sham, Phys. Rev. 140, A1133 (1965).

[27] J. P. Perdew and A. Zunger, Phys. Rev. B 23, 5048-5079 (1981).

[28] D. M. Ceperley and B. J. Alder, Phys. Rev. Lett. 45, 566569 (1980).

[29] S. Fahy, X. W. Wang, and S. G. Louie, Phys. Rev. B 42, 3503 (1990).

[30] A. J. Williamson et al., Phys. Rev. B 53, 9640 (1996).

[31] S. Fahy, X. W. Wang, and S. G. Louie, Phys. Rev. Lett. 65, 1478 (1990).

[32] H. J. Monkhorst and J.D. Pack, Phys. Rev. B 13, 5188 (1976).

[33] P. M. Platzman and N. Tzoar, Phys. Rev. 139, 410 (1965).

[34] P. Eisenberger and P. M. Platzman, Phys. Rev. A 2, 415 (1970).

[35] L. Lam and P. M. Platzman, Phys. Rev. B 9, 5122 (1974).

[36] G. Ortiz and P. Ballone, Phys. Rev. B 50, 1391 (1994). 